\title{
Resistance training improves isokinetic strength and metabolic syndrome-related phenotypes in postmenopausal women
}

This article was published in the following Dove Press journal:

Clinical Interventions in Aging

7 August 2015

Number of times this article has been viewed

\author{
Pedro Ferreira Alves \\ Oliveira' \\ André Bonadias Gadelha ${ }^{2}$ \\ Rafael Gauche ${ }^{2}$ \\ Flávio Macedo Lahud Paiva ${ }^{2}$ \\ Martim Bottaro ${ }^{2}$ \\ Lauro C Vianna ${ }^{2}$ \\ Ricardo Moreno Lima² \\ 'Department of Physical Education, \\ Instituto Federal de Brasília, ${ }^{2}$ College \\ of Physical Education, University of \\ Brasília, Brasília, DF, Brazil
}

Purpose: To examine the effects of resistance training (RT) on metabolic syndrome-related phenotypes in postmenopausal women.

Patients and methods: Twenty-two postmenopausal women (65.0 \pm 4.2 years) underwent 12 weeks of whole body progressive training with intensity prescribed based on rating of perceived exertion. Dominant knee extension strength was assessed using an isokinetic dynamometer before and after the intervention. Moreover, all volunteers had blood samples collected for lipid profile, glycemic control, and C-reactive protein analyses. Waist circumference and arterial blood pressure were also measured at baseline and after the training period. Student's $t$-tests for paired samples and repeated measures ANOVA were used to compare dependent variables, and statistical significance was set at $P<0.05$.

Results: Isokinetic muscle strength significantly increased $(P<0.01)$ with training. It was observed that waist circumference as well as total and low-density lipoprotein cholesterol levels significantly decreased with training $(P<0.01)$. Total cholesterol/high-density lipoprotein cholesterol ratio, an important marker of cardiovascular disease incidence, was also significantly reduced (from $3.91 \pm 0.91$ to $3.60 \pm 0.74 ; P<0.01$ ) after the program. Blood glucose, basal insulin, and homeostatic model assessment of insulin resistance were also significantly reduced $(P<0.01)$. No significant alterations were observed for resting blood pressure, triglycerides, or C-reactive protein.

Conclusion: Based on the observed results, it can be concluded that a 12-week progressive RT program, besides increasing isokinetic muscle strength, induces beneficial alterations on metabolic syndrome-related phenotypes in postmenopausal women. These findings highlight this mode of exercise as an important component of public health promotion programs for aged women. RT improves isokinetic strength and metabolic syndrome-related phenotypes in postmenopausal women.

Keywords: strength training, cardiometabolic risk, OMNI-RES, aging

\section{Introduction}

Resistance training (RT) has been widely studied in individuals with advanced age, given its well-documented effects in preventing and restoring the decline in muscle mass and strength (ie, sarcopenia). ${ }^{1-3}$ Sarcopenia has been linked to a variety of negative outcomes, and the benefits of the RT introduce its practice in several guidelines from important institutions involved in global physical activity programs for older adults. ${ }^{4-8}$ However, although the effects of RT in improving muscle strength and function are well recognized, its role in managing cardiometabolic risk factors has only lately attracted attention of scientific community. ${ }^{9,10}$ Noteworthy, a plethora of epidemiological studies demonstrate that cardiovascular and metabolic risks
Correspondence: Ricardo Moreno Lima Faculdade de Educação Física, Universidade de Brasília (UnB), Campus Universitário Darcy Ribeiro, Brasília 70910-900, DF, Brazil

Tel +556181099444

$\mathrm{Fax}+556131072500$

Email ricardomoreno@unb.br 
predominantly affect older individuals. ${ }^{4,11,12}$ Despite this, it is still unclear whether RT program is an effective tool against cardiometabolic risk factors in this population.

The clustering of cardiometabolic risk factors has been referred to as metabolic syndrome, a complex disorder with important implications of health care costs and considered a worldwide epidemic. ${ }^{13,14}$ The prevalence of metabolic syndrome has increased and is dependent on sex and age. For example, there is a higher incidence in postmenopausal women, who after menopause undergo significant adverse changes in body composition, including loss of fat-free mass, increases in fat mass, and redistribution of fat from the periphery to the central region of the body. Furthermore, menopause is associated with greater reductions in muscle mass and strength, especially among sedentary women. ${ }^{15}$ All together, the postmenopausal period itself is an independent risk factor for the development of this syndrome. ${ }^{16}$ Given this background, it becomes important to develop strategies to effectively control and improve metabolic syndrome-related phenotypes in this population, including in subjects who do not present this syndrome.

Metabolic syndrome-related phenotypes include abnormal lipid profile (ie, elevated triglycerides, total cholesterol, and decreased high-density lipoprotein [HDL]), elevation of arterial blood pressure (BP), and impaired glucose homeostasis, while abdominal obesity has received increasing attention as the pivotal manifestation of this syndrome. In fact, waist circumference (WC) has been consistently associated with a variety of risk markers. ${ }^{4}$ Recently, increases in the concentration of several inflammatory biomarkers have been added to metabolic syndrome entity. ${ }^{5,13}$ It should be pointed out that C-reactive protein (CRP) concentration, like other inflammatory indexes, increases with advancing age and is associated with incident cardiovascular disease and mortality. ${ }^{17}$

Management of metabolic syndrome-related traits is clearly warranted, being change in lifestyle the first-line of prevention and treatment. Indeed, a variety of reports have shown that physical activity in general promotes favorable effects on many cardiovascular and metabolic risk factors. ${ }^{4,8,18-20}$ Recently, RT has also been examined with respect to positively impact cardiometabolic status; ${ }^{1,2,21}$ however, more studies are needed to define such effects. Thus, the purpose of the present study was to examine the effects of 12 weeks of RT on metabolic syndrome-related phenotypes in postmenopausal women without the syndrome.

\section{Materials and methods}

\section{Participants}

After exclusion criteria were applied, a total of 30 postmenopausal women took part in the present study. Volunteers were recruited through flyers, banners, phone calls, and visits to centers of leisure for elderly people, hospitals, and churches. All participants answered a face-to-face questionnaire addressing medical history, medication use, and comorbidities. Exclusion criteria used were as follows: being engaged in regular physical exercises for the last 3 months, hormone therapy, smoking, age lower than 60 years and higher than 80 years, metabolic or endocrine disorders known to affect the musculoskeletal system, uncontrolled hypertension or type 2 diabetes, coronary artery disease, walk only with assistance, and present any medical condition that would be aggravated with the study protocol. Twenty-two volunteers successfully attended measurements and training, and thus composed the final sample of the study (mean age of $65.0 \pm 4.2$ years; $15 \pm 6.31$ years of menopause). Reasons for noncompliance included injuries not related to training $(\mathrm{n}=2)$, address alteration $(n=1)$, and frequency to training sessions lower than $75 \%(n=5)$. All volunteers underwent physician evaluation before baseline testing procedures to ensure exclusion criteria identification. The research protocol was approved by the University of Brasilia Ethics Committee. All participants signed an informed consent form containing the objectives and procedures as well as possible risks and benefits related to the study design.

\section{Metabolic and cardiovascular risk}

BP was measured two times following 5 and 10 minutes of rest, respectively, using an oscillometric automated device (BP 3AC1-1 PC; Microlife, Widnau, Switzerland) with volunteers in the seated position. The mean values of systolic and diastolic BP from the two measurements were considered for analyses. Within-subjects coefficient of variation for the used device was $6.0 \%$ and $5.7 \%$ for systolic and diastolic BP, respectively. WC was measured using an inextensible tape (Sanny Anthropometric Medical, São Bernardo do Campo, Brazil) at the midpoint between the last rib and iliac crest. Blood samples were collected following an overnight fast (12 hours). Biological material was immediately moved to laboratory analysis for glucose, CRP, lipid profile, and insulin. In addition, homeostatic model assessment of insulin resistance (HOMA-IR) was calculated based on the product of plasma insulin concentration $(\mu \mathrm{UI} / \mathrm{mL})$ and plasma glycemic concentration $(\mathrm{mmol} / \mathrm{L})$ divided by $22.5 .^{22}$ Briefly, triglycerides, cholesterol, and subfractions as well as blood glucose were measured using an enzymatic colorimetric method, processed in an Autohumalyzer A5 (Human, Wiesbaden, Germany). Plasma insulin concentration was assayed using the Automated Chemiluminescence System ACS-180 (Ciba-Corning Diagnostics Corp., Medfield, Massachusetts, USA, 1995). CRP was 
examined through a turbidimetric method. Inter- and intra-assay coefficients of variation for blood variables determination did not exceed $4.5 \%$. Specifically for blood biochemistry, measurements were performed in three time points as follows: 12 weeks before training initiation, before RT, and after RT.

\section{Isokinetic muscle torque}

Dominant knee extensor isokinetic peak torque (PT) was evaluated using the Biodex System 3 dynamometer (Biodex Medical Systems, Inc., Shirley, NY, USA). All measurements were carried out by the same trained technician according to procedures described elsewhere. ${ }^{2}$ After a familiarization trial consisting of two sets of ten repetitions, the testing protocol consisted of four sets of four knee extensor contractions (set 1 and 2 at $60 \%$ s, set 3 and 4 at $180 \%$ s) with 60 seconds rest between sets. The recorded value was the single muscle contraction that elicited the highest PT throughout the protocol in each velocity, which is expressed in absolute values $(\mathrm{N} \cdot \mathrm{m})$. Participants were fully informed about test procedures and asked to perform the movement with their maximal strength while verbal encouragement was offered. Calibration of the equipment was performed according to the manufacturer's specifications before every testing session.

\section{RT program}

Volunteers took part in a 12-week RT program and underwent physician evaluation before participation. Before the training period, participants underwent 1 week of three familiarization sessions to ensure proper execution of technique. The training program was performed three times per week during 12 weeks. The protocol aimed to work out major muscle groups and involved the following exercises: chest press, knee extension, hamstrings curl, leg press, hip abduction, seated row, shoulder abduction, and plantar flexion (Rotech Fitness Equipment, Goiania, Brazil). Training loads were monitored and adjusted using the OMNI-RES. ${ }^{23}$ The program followed a linear progressive fashion, with training loads at 6 point (somewhat hard) during the first 4 weeks, 7 point (between somewhat hard and hard) during following 4 weeks, and 8 point (hard) over the remaining 4 weeks, with repetitions, respectively, decreasing from 12, 10, and 8. Each exercise was performed in three sets with approximately 1 minute rest between sets. Except for the implementation of the RT program, all volunteers were asked to maintain their habitual routine throughout the study. Moreover, all training sessions were closely supervised by at least three qualified professionals.

\section{Statistical analyses}

Normal distribution of data was examined using the ShapiroWilk test. Values were expressed as mean and standard deviation or standard error. Student's $t$-tests for paired samples were used to compare dependent variables between preintervention and postintervention, while repeated measures ANOVA was performed to compare blood variables 12 weeks before training initiation, pre training, and post training. Adequate sample size was calculated for ANOVA with repeated measures (within factor), applying a desired power of 0.80 and an $\alpha$ error of 5\%. Using the effect size of 0.30 (Cohen's $d$ for within-subject designs), the minimum sample size was determined to be 21 subjects. ${ }^{24}$ Also, Cohen's $d$ effect size was calculated for each comparison. Significance level was set at $P<0.05$, and all analyses were performed using the Statistical Package for the Social Sciences version 18.0.

\section{Results}

A total of $54.5 \%$ of the volunteers reported controlled hypertension, $22.7 \%$ osteoporosis, $13 \%$ hyperlipidemia, and $9.1 \%$ controlled type 2 diabetes mellitus, but none of them had metabolic syndrome. Medication treatment remained unchanged throughout the study period. Comparisons of anthropometric, $\mathrm{BP}$, triglycerides, CRP, and strength variables between preintervention and postintervention are presented in Table 1. It can

Table I Comparison of anthropometric, blood pressure, triglycerides, C-reactive protein, and strength variables between preintervention and postintervention $(n=22)$

\begin{tabular}{llll}
\hline Variable & Preintervention & Postintervention & Effect size \\
\hline Body mass $(\mathrm{kg})$ & $68.9 \pm 13.3$ & $68.1 \pm 13.2^{*}$ & 0.054 \\
Body mass index $\left(\mathrm{kg} / \mathrm{m}^{2}\right)$ & $28.4 \pm 5.0$ & $27.9 \pm 4.8^{*}$ & 0.094 \\
Waist circumference $(\mathrm{cm})$ & $98.3 \pm 13.2$ & $94.6 \pm 12.6^{*}$ & 0.283 \\
Systolic blood pressure $(\mathrm{mmHg})$ & $127.9 \pm 10.1$ & $125.5 \pm 15.5$ & 0.180 \\
Diastolic blood pressure $(\mathrm{mmHg})$ & $75.6 \pm 6.7$ & $76.5 \pm 9.2$ & 0.106 \\
C-reactive protein $(\mathrm{mg} / \mathrm{dL})$ & $0.26 \pm 0.4$ & $0.22 \pm 0.2$ & 0.131 \\
Peak torque $60 \% \mathrm{~s}(\mathrm{~N} \cdot \mathrm{m})$ & $90.3 \pm 24.1$ & $101.9 \pm 21.4^{*}$ & 0.512 \\
Peak torque $180 \% \mathrm{~s}(\mathrm{~N} \cdot \mathrm{m})$ & $61.8 \pm 16.8$ & $71.5 \pm 16.3^{*}$ & 0.588 \\
\hline
\end{tabular}

Note: *Significantly different in relation to baseline $(P<0.05)$.

Abbreviation: $\mathrm{N} \cdot \mathrm{m}$, newton meter. 

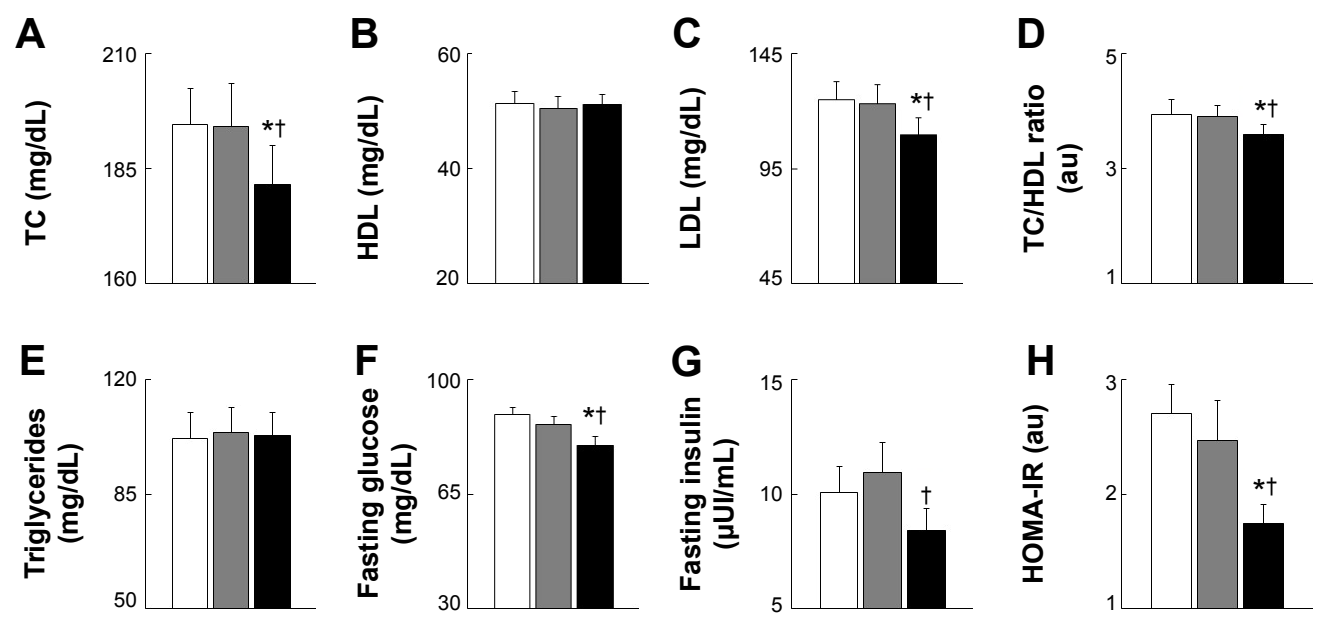

12 wk before training

Pre training

Post training

Figure I Mean values and standard error for variables related to lipid profile and glycemic control 12 weeks before training initiation and before and after the training period.

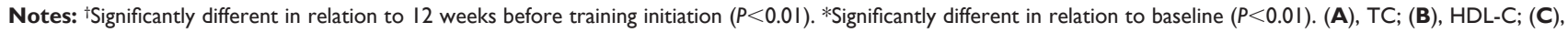
LDL-C; (D), TC/HDL ratio; (E), triglycerides; (F), fasting blood glucose; (G), fasting blood insulin; and (H), HOMA-IR.

Abbreviations: TC, total cholesterol; HDL-C, high-density lipoprotein cholesterol; LDL-C, low-density lipoprotein cholesterol; HOMA-IR, homeostatic model of insulin resistance; au, arbitrary unit.

be observed that body mass, BMI, and WC were significantly decreased after the training period $(P<0.05, P<0.05$ and $P<0.01$, respectively). Moreover, isokinetic PT significantly increased $(P<0.01)$ with training for the two evaluated velocities (ie, $60^{\circ} / \mathrm{s}$ and $180 \%$ s). No significant alterations were observed for systolic and diastolic BP or CRP.

Figure 1 presents mean values for variables related to lipid profile and glycemic control 12 weeks before training initiation and before and after the training period. It was observed that total and low-density lipoprotein (LDL) cholesterol, as well as total cholesterol divided by HDL, significantly decreased $(P<0.01)$, all with medium effect sizes (Cohen's $d$ varying from 0.307 to 0.374 ). Also, blood glucose, basal insulin, and HOMA-IR were significantly reduced $(P<0.01)$ after the intervention (Cohen's $d$ varying from 0.475 to 0.567$)$. No significant alterations were noted for triglycerides or HDL cholesterol.

\section{Discussion}

The main findings of the present study were that 12 weeks of RT, besides the well-described improvements in muscle strength, induced beneficial alterations on several cardiometabolic risk factors in postmenopausal women. In particular, significant reductions were noted for WC, total cholesterol, LDL cholesterol, blood glucose, basal insulin, and HOMA-IR. The training protocol characteristics were similar to several studies available in the literature and followed the American College of Sports Medicine's recommendations, ${ }^{9,10,25}$ with training load prescribed based on rate of perceived exertion using the OMNI-RES, an instrument that had been previously validated. ${ }^{23,26}$ Importantly, volunteers showed significant increases in muscle strength assessed through an isokinetic dynamometer, a method that has become a gold standard and is well known for its validity in both clinical and research settings. Although volunteers did not have the metabolic syndrome, the postmenopausal period itself is an independent risk factor for the development of this condition, ${ }^{15}$ and thus, interventions effective in improving metabolic profile are meaningful in this population. The observations indicating the role of RT on the prevention of metabolic syndrome have important clinical implications and reinforce RT as a relevant component of health promotion programs designed to older women.

The results of the present study are in agreement with those of Dunstan et $\mathrm{a}^{10}$ who reported a significant reduction in body mass $(-1.8 \pm 2.0 \mathrm{~kg})$ and $\mathrm{WC}(3.8 \pm 3.5 \mathrm{~cm})$ in older patients with type 2 diabetes. However, Dunstan et a ${ }^{10}$ examined RT in conjunction with caloric restriction and thus did not address the independent effects of training. Literature in this regard is still controversial, with Joseph et $\mathrm{al}^{27}$ reporting that following 12 weeks of RT, older men had significant decreases in adiposity indexes, whereas the older women demonstrated no change. The effects of RT on lipid profile are not consensual. The present investigation 
observed significant reductions in total (approximately 6.5\%) and LDL (approximately 11\%) cholesterols as well as in total cholesterol/HDL ratio, a better indicative of cardiovascular diseases, results that mirror those from Prabhakaran et $\mathrm{al}^{25}$ in healthy sedentary premenopausal women (mean age 28 years). These results are also in agreement with data presented by Gavin et $\mathrm{al}^{21}$ who presented that cholesterol reduced with RT but not with aerobic exercises. However, other studies failed to observe significant alterations. ${ }^{9,21}$

Research on the effects of resistance exercise on diabetes management has only recently received greater attention. The present study provide the insight that 12 weeks of progressive RT with prescription based on rate of perceived exertion independently promotes improvements on glycemic control of healthy sedentary postmenopausal women, with decline in plasma glucose and insulin concentration as well as in HOMA-IR score. Impaired insulin sensitivity is closely related to aging, but most studies that examined RT effects in the elderly were limited to type 2 diabetic patients. ${ }^{8,10}$ In this regard, only two of the present study volunteers had type 2 diabetes who when removed from analyses did not change results (data not shown), suggesting the role of RT on prevention of this condition. Several ${ }^{10,28}$ but not all studies ${ }^{29}$ also found that RT reduced fasting plasma glucose and insulin concentration. In conjunction with reports showing that 12 weeks of RT promoted significant reduction in glycated hemoglobin $(-7.4 \%$ compared to baseline), ${ }^{10}$ a maker of glucose concentration over prolonged periods of time, available evidences present RT as an important component of exercise programs for type 2 diabetes. ${ }^{8}$ The present training protocol, however, did not significantly alter HDL cholesterol or triglycerides. These results are in agreement with the study by Dunstan et a ${ }^{10}$ who suggest that a longer period of training along with changes in body weight may be necessary to promote significant alterations on these variables after RT.

We recognize limitations in the present study. The experimental design did not include a control group. However, blood biochemistry variables were presented 12 weeks before training initiation (as a time-control) and before and after RT, and analyses showed that significant alterations were only observed when comparing pre training and post training. Also, positive changes are strongly unexpected without any sort of intervention in this population, and thus, absence of control group is not rare on existing related articles. ${ }^{27,30,31}$ It is also important to mention that there was no nutritional control, but participants were instructed not to change their habitual routine, including physical activity and eating behaviors. Of note, this recommendation was reinforced every single session during the intervention period and was checked with volunteers as an attempt to minimize possible influences on the observed results.

\section{Conclusion}

Based on the observations presented here, it can be concluded that a 12-week progressive RT program besides promoting increases in isokinetic muscle strength induces beneficial alterations on metabolic syndrome-related phenotypes in postmenopausal women. Specifically, the benefits were related to WC decrease; positive alterations on lipid profile, including reductions in total and LDL cholesterols as well as in TC/HDL ratio, an important marker of cardiovascular diseases; and improvements on glycemic control, with decline on fasting blood glucose, insulin concentration, and HOMA-IR. No alterations, however, were observed for triglycerides, HDL, resting BP, or CRP, being the latter a marker of inflammation. These findings indicate the role of RT on the prevention of metabolic syndrome and highlight this mode of exercise as an important component of physical activity programs for elderly people.

\section{Acknowledgments}

We thank the participants of the study and the Sabin Laboratory, which allowed analyses of blood variables. This work was supported by National Council for Scientific and Technological Development grants 487622/2012-0 and 307630/2013-7 by University of Brasília.

\section{Disclosure}

The authors report no conflicts of interest in this work.

\section{References}

1. Pillard F, Laoudj-Chenivesse D, Carnac G, et al. Physical activity and sarcopenia. Clin Geriatr Med. 2011;27(3):449-470.

2. Lima RM, Leite TK, Pereira RW, Rabelo HT, Roth SM, Oliveira RJ. $\mathrm{ACE}$ and ACTN3 genotypes in older women: muscular phenotypes. Int J Sports Med. 2011;32(1):66-72.

3. Johnston AP, De Lisio M, Parise G. Resistance training, sarcopenia, and the mitochondrial theory of aging. Appl Physiol Nutr Metab. 2008;33(1): 191-199.

4. dos Santos EP, Gadelha AB, Safons MP, Nobrega OT, Oliveira RJ, Lima RM. Sarcopenia and sarcopenic obesity classifications and cardiometabolic risks in older women. Arch Gerontol Geriatr. 2014;59(1): $56-61$.

5. Stensvold D, Tjønna AE, Skaug EA, et al. Strength training versus aerobic interval training to modify risk factors of metabolic syndrome. $J \mathrm{Appl}$ Physiol. 2010;108(4):804-810.

6. Williams MA, Haskell WL, Ades PA, et al; American Heart Association Council on Clinical Cardiology, American Heart Association Council on Nutrition, Physical Activity, and Metabolism. Resistance exercise in individuals with and without cardiovascular disease: 2007 update a scientific statement from the American Heart Association Council on Clinical Cardiology and Council on Nutrition, Physical Activity, and Metabolism. Circulation. 2007;116(5):572-584. 
7. American College of Sports Medicine; Chodzko-Zajko WJ, Proctor DN, et al. American College of Sports Medicine position stand. Exercise and physical activity for older adults. Med Sci Sports Exerc. 2009;41(7): 1510-1530.

8. Colberg SR, Sigal RJ, Fernhall B, et al; American Diabetes Association. Exercise and type 2 diabetes the American College of Sports Medicine and the American Diabetes Association: joint position statement. Diabetes Care. 2010;33(12):e147-e167.

9. Bateman LA, Slentz CA, Willis LH, et al. Comparison of aerobic versus resistance exercise training effects on metabolic syndrome (from the studies of a targeted risk reduction intervention through defined exercise - STRRIDE-AT/RT). Am J Cardiol. 2011;108(6):838-844.

10. Dunstan DW, Daly RM, Owen N, et al. High-intensity resistance training improves glycemic control in older patients with type 2 diabetes. Diabetes Care. 2002;25(10):1729-1736.

11. Manzato E, Romanato G, Zambon S, et al. Metabolic syndrome and cardiovascular disease in the elderly: the Progetto Veneto Anziani (Pro. VA) Study. Aging Clin Exp Res. 2008;20(1):47-52.

12. Ishii S, Tanaka T, Akishita M, Ouchi Y, Tuji T, Iijima K. Metabolic syndrome, sarcopenia and role of sex and age: cross-sectional analysis of Kashiwa cohort study. PLoS One. 2014;9(11):e112718.

13. Kassi E, Pervanidou P, Kaltsas G, Chrousos G. Metabolic syndrome: definitions and controversies. BMC Med. 2011;9:48.

14. Ford ES, Li C, Zhao G, Pearson WS, Mokdad AH. Prevalence of the metabolic syndrome among U.S. adolescents using the definition from the International Diabetes Federation. Diabetes Care. 2008;31(3): 587-589.

15. Vianna LC, Oliveira RB, Araújo CGS. Age-related decline in handgrip strength differs according to gender. J Strength Cond Res. 2007;21(4): 1310-1314

16. Cho GJ, Lee JH, Park HT, et al. Postmenopausal status according to years since menopause as an independent risk factor for the metabolic syndrome. Menopause. 2008;15(3):524-529.

17. Cesari M, Onder G, Zamboni V, et al. C-reactive protein and lipid parameters in older persons aged 80 years and older. $J N H A .2009 ; 13(7)$ : 587-594.

18. Wu L, Chen R, Ma D, Zhang S, Walton-Moss B, He Z. Effects of lifestyle intervention improve cardiovascular disease risk factors in community-based menopausal transition and early postmenopausal women in China. Menopause. 2014;21(12):1263-1268.

19. Zhang J, Chen G, Lu W, et al. Effects of physical exercise on health-related quality of life and blood lipids in perimenopausal women: a randomized placebo-controlled trial. Menopause. 2014;21(12):1269-1276.
20. Lee J-A, Kim J-W, Kim D-Y. Effects of yoga exercise on serum adiponectin and metabolic syndrome factors in obese postmenopausal women. Menopause. 2012;19(3):296-301.

21. Gavin C, Sigal RJ, Cousins M, et al; Diabetes Aerobic and Resistance Exercise (DARE) trial investigators. Resistance exercise but not aerobic exercise lowers remnant-like lipoprotein particle cholesterol in type 2 diabetes: a randomized controlled trial. Atherosclerosis. 2010;213(2): 552-557.

22. Matthews D, Hosker J, Rudenski A, Naylor B, Treacher D, Turner R. Homeostasis model assessment: insulin resistance and $\beta$-cell function from fasting plasma glucose and insulin concentrations in man. Diabetologia. 1985;28(7):412-419.

23. Robertson RJ, Goss FL, Rutkowski J, et al. Concurrent validation of the OMNI perceived exertion scale for resistance exercise. Med Sci Sports Exerc. 2003;35(2):333-341.

24. Lakens D. Calculating and reporting effect sizes to facilitate cumulative science: a practical primer for t-tests and ANOVAs. Front Psychol. 2013;4:863.

25. Prabhakaran B, Dowling EA, Branch JD, Swain DP, Leutholtz BC. Effect of 14 weeks of resistance training on lipid profile and body fat percentage in premenopausal women. Br J Sports Med. 1999;33(3): 190-195.

26. Gearhart RF Jr, Lagally KM, Riechman SE, Andrews RD, Robertson RJ. Safety of using the adult OMNI resistance exercise scale to determine 1-RM in older men and women. Percept Mot Skills. 2011;113(2): 671-676.

27. Joseph LJ, Davey SL, Evans WJ, Campbell WW. Differential effect of resistance training on the body composition and lipoprotein-lipid profile in older men and women. Metabolism. 1999;48(11):1474-1480.

28. Misra A, Alappan NK, Vikram NK, et al. Effect of supervised progressive resistance-exercise training protocol on insulin sensitivity, glycemia, lipids, and body composition in Asian Indians with type 2 diabetes. Diabetes Care. 2008;31(7):1282-1287.

29. Reis Filho AD, Vitto JPP, Vieira RC Jr, Voltarelli FA. Blood glucose analyzes after 12 weeks of strength training in women. R. bras. Ci. e Mov. 2013;21(3):7.

30. Yamada M, Arai H, Uemura K, et al. Effect of resistance training on physical performance and fear of falling in elderly with different levels of physical well-being. Age Ageing. 2011;40(5):637-641.

31. Phillips B, Williams J, Atherton P, et al. Resistance exercise training improves age-related declines in leg vascular conductance and rejuvenates acute leg blood flow responses to feeding and exercise. $J$ Appl Physiol. 2012;112(3):347-353.
Clinical Interventions in Aging

\section{Publish your work in this journal}

Clinical Interventions in Aging is an international, peer-reviewed journal focusing on evidence-based reports on the value or lack thereof of treatments intended to prevent or delay the onset of maladaptive correlates of aging in human beings. This journal is indexed on PubMed Central, MedLine,
Dovepress

CAS, Scopus and the Elsevier Bibliographic databases. The manuscript management system is completely online and includes a very quick and fair peer-review system, which is all easy to use. Visit http://www.dovepress. com/testimonials.php to read real quotes from published authors. 